Abstract

\title{
Particle Path Integrals on Maximally Symmetric Spaces and Type-A Trace Anomalies ${ }^{+}$
}

\author{
Olindo Corradini ${ }^{1,2}$ \\ 1 Dipartimento di Scienze Fisiche, Informatiche e Matematiche, \\ Università degli Studi di Modena e Reggio Emilia, 41125 Modena MO, Italy; olindo.corradini@unimore.it \\ 2 Istituto Nazionale di Fisica Nucleare (INFN), 40126 Bologna BO, Italy \\ + Presented at Symmetry 2017-The First International Conference on Symmetry, Barcelona, Spain, \\ 16-18 October 2017.
}

Published: 12 January 2018

Particle path integrals in curved spaces can be employed to compute trace anomalies in quantum field theories, and more generally to study properties of quantum fields coupled to gravity. Their construction in arbitrary coordinates is well understood, and known to require the use of a regularization scheme. In the present talk, we elaborate on an old proposal that focuses on the construction of the path integral by using Riemann normal coordinates. The main method's conjecture is that curvature effects are taken care of by a scalar effective potential, so that the particle Lagrangian is reduced to that of a linear sigma model interacting with the effective potential. We show the explicit validity of the simplified path integral on maximally symmetric spaces, and test it to compute heat kernel coefficients and type-A trace anomalies for a scalar field in arbitrary dimensions up to $d=12$. The results agree with those expected, which are reproduced with great efficiency and extended to higher orders. Finally, we point out that this simplified path integral might be of further use in worldline applications, though its application on spaces of arbitrary geometry remains unclear.

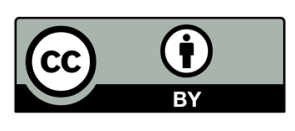

(c) 2018 by the author. Licensee MDPI, Basel, Switzerland. This article is an open access article distributed under the terms and conditions of the Creative Commons Attribution (CC BY) license (http://creativecommons.org/licenses/by/4.0/). 